\title{
SUCCESSFUL REPAIR OF POSTINFARCTION LEFT VENTRICULAR FREE WALL RUPTURE: NEW STRATEGY WITH HYPOTHERMIC PERCUTANEOUS CARDIOPULMONARY BYPASS
}

\author{
Tetsuo Sakakibara, MD, ${ }^{\text {a }}$ Ryousuke Matsuwaka, MD,${ }^{\text {a }}$ Hideo Shintani, MD, ${ }^{\text {a }}$ Akihiko Yagura, MD, ${ }^{\text {a }}$
} Takahiro Yamaguchi, MD, ${ }^{\mathrm{a}}$ Atsushi Hirayama, MD ${ }^{\mathrm{b}}$ and Kazuhisa Kodama, MD, ${ }^{\mathrm{b}}$ Osaka, Japan

Despite recent advances in intensive care for coronary artery disease, left ventricular free wall rupture still remains as a fatal complication of acute myocardial infarction. Especially for the blowout type of left ventricular free wall rupture, reports of survival without any sequela are rare. Here we present a successfully repaired case of the blowout type of left ventricular free wall rupture with percutaneous cardiopulmonary bypass (PCPB) and deep hypothermia.

A 78-year-old woman was admitted with sustained chest pain and dyspnea. Emergency coronary angiography showed that the infarct-related vessel was a right coronary artery, and thrombolytic therapy was therefore administered. When this intervention was completed, the patient suddenly lost consciousness and then had ventricular fibrillation. While the patient was maintained on conventional cardiopulmonary resuscitations, the PCPB system consisting of a centrifugal pump (Sarns 7850; Sarns, 3M Health Care, Ann Arbor, Mich.) and a heparin-coated membrane oxygenator (Maxima; Medtronic, Inc., Cardiopulmonary Division, Anaheim, Calif.), was quickly set up. The $15 \mathrm{~F}$ and $17 \mathrm{~F}$ heparin-coated thin-wall cannulas (BioMedicus; Medtronic) were inserted into the left femoral artery and vein, respectively, after systemic heparinization $(1 \mathrm{mg} / \mathrm{kg}$ ). Venoarterial bypass support was started 20 minutes after cardiac arrest. The left side of the chest cavity was then exposed through a fifth intercostal anterolateral incision. The pericardial space was found to be filled with blood. After pericardiectomy, which corrected the cardiac tamponade, the heart started beating spontaneously. Free bleeding from a left ventricular inferior muscular tear was detected. During these surgical procedures, the blood temperature was cooled down to $20^{\circ} \mathrm{C}$ with the heat exchanger incorporated in the oxygenator, resulting in hypothermic fibrillatory arrest of the heart. The left ventricular rupture was repaired with double 3-0 monofilament mattress sutures reinforced by felt pledgets. After completion of rewarming, the patient was weaned from the bypass with the aid of intraaortic balloon pumping and inotropic support. PCPB time was 114 minutes. Even though the patient's postoperative recovery was complicated by chronic obstructive lung disease, which delayed the removal of ventilatory support, she was discharged from the hospital on the 80th postoperative day without any complications.

From the Divisions of Cardiovascular Surgery ${ }^{\mathrm{a}}$ and Cardiology, ${ }^{\mathrm{b}}$

Cardiovascular Center, Osaka Police Hospital, Osaka, Japan.

Accepted for publication June 7, 1995.

J THORAC CARDIovasc Surg 1996;111:276.

Copyright (C) 1996 by Mosby-Year Book, Inc.

$0022-5223 / 96 \$ 5.00+0 \quad \mathbf{1 2 / 5 4 / 6 6 8 3 1}$
PCPB is now becoming a standard resuscitative modality for the treatment of severe cardiogenic shock. ${ }^{1}$ Our previous strategy was to use PCPB as a bridge to an operation performed under standard bypass performed in the operating room. ${ }^{2}$ This technique was consistent with other reports of how left ventricular rupture was handled. ${ }^{3}$ There are several advantages in our new strategy. Left thoracotomy under PCPB support, without transferring the patient to the operating room, is the quickest approach for the immediate correction of cardiac tamponade. Deep hypothermia is beneficial to both myocardium and brain. Hypothermic fibrillatory arrest, which can be induced with PCPB, is a simple and reliable arrest method during emergency cardiac procedures. ${ }^{4}$ Cerebral hypothermia after conventional cardiopulmonary resuscitation may enhance the neurologic recovery. ${ }^{5}$ Reduction of the optimal cardiopulmonary bypass flow rate by hypothermia may be beneficial when adequate bypass flow cannot be obtained at normothermia because of possible hypovolemia in a patient in shock. To our best knowledge, this is the first report to describe the use of hypothermic PCPB for successful repair of postinfarction left ventricular free wall rupture. Even though this is only one case report, we believe that our new strategy for left ventricular free wall rupture may bring great improvement to this challenging area of cardiovascular surgery.

\section{REFERENCES}

1. Mooney MR, Arom KV, Joyce LD, et al. Emergency cardiopulmonary bypass support in patients with cardiac arrest. J Thorac CARdiovasc SuRg 1991;101:450-4.

2. Masai T, Sakakibara T, Watanabe S, Kodama K, Kaneko M, Matsuda H. A case report of successful surgical treatment following the emergency circulatory assist by percutaneous cardiopulmonary support system for acute post infarction left ventricular free wall rupture [Japanese]. J Jpn Assoc Thorac Surg 1992;40: 86-90.

3. Scholz KH, Werner GS, Schorn B, Baryalei MM, Kreuzer H, Figulla HR. Post infarction left ventricular rupture: successful surgical intervention after percutaneous cardiopulmonary support during mechanical resuscitation. Am Heart J 1994;127:210-1.

4. Akins CW. Early and late results following emergency isolated myocardial revascularization during hypothermic fibrillatory arrest. Ann Thorac Surg 1987;43:131-7.

5. Sterz F, Safar P, Tisherman S, Radovsky A, Kuboyama $\mathrm{K}$, Oku K. Mild hypothermic cardiopulmonary resuscitation improves outcome after prolonged cardiac arrest in dog. Crit Care Med 1991;19:379-89. 